\title{
A PROOF OF A CONJECTURE OF VANDIVER
}

\section{N. HERSTEIN}

The Wedderburn theorem that every finite division ring is commutative has been extended by several authors [1]. ${ }^{1}$ Vandiver, in his paper The p-adic representation of rings [2] conjectured the following generalization of Wedderburn's theorem: every finite, non-commutative ring contains an element which is a divisor of zero and is not in the centrum.

In this paper we give a short and simple proof of this conjecture. We also exhibit one generalization of it which was pointed out to us by the referee.

THEOREM. A finite non-commutative ring contains an element which is a divisor of zero and is not in the centrum of the ring.

Proof. Let $R$ be the ring, $C$ its centrum and $N$ its radical. If every element of $R$ is a divisor of zero, then the theorem is trivially true. So we may assume that $D$, the set of elements in $R$ which are not divisors of zero, is not empty. We claim that $D$ is a multiplicative group. For if $x, y, z \in D$, then $x y \in D$; and if $x y=z y$ or $y x=y z$, then $x=z$. Hence, since $D$ is finite, it forms a group under multiplication, and so contains a two-sided unit element, 1 . We assert that 1 is a unit element for $R$. For if $r \in R$, then it can be written as $r=x d$ where $d \in D$ (since all the left multiples of $d$ are distinct and so cover all of $R)$. Thus $r 1=(x d) 1=x(d 1)=x d=r$. Similarly 1 is a left unit.

Now if $\dot{R}$ contains a nonzero idempotent $e \neq 1$, then for all $x \in R$, $x e(1-e)=x(1-e) e=0$, and so if the theorem were false, both $x e$ and $x(1-e)$ would be in $C$; hence $x e+x(1-e)=x$ would be in $C$. And thus $R=C$, contradicting that $R$ is non-commutative. Hence we may assume that 1 is the only nonzero idempotent in $R$.

Since $1 \notin N, N \neq R$. Let $x \notin N$. Then $R x$ is a non-nilpotent left ideal, and so contains a nonzero idempotent [3]; thus $R x$ contains 1 . That is, if $X \notin N$, then $x$ is regular. Hence $R-N$ is a finite division ring, which by the Wedderburn theorem is commutative. But the multiplicative group of a finite field is cyclic; that is, there exists an $\bar{a}$ $\in R-N$ such that for every $\bar{x} \neq 0$ in $R-N$, there exists an integer $s$ so that $\bar{x}=\bar{a}^{s}$. Let $a \in R$ map an $\bar{a} \in R-N$. Whence for every $x \in R$, $x \notin N$ we can find an integer $S$ so that $a^{s}-x \in N$. If $N$ is not contained

Presented to the Society, February 26, 1949; received by the editors February 17, 1949 and, in revised form, April 25, 1949.

1 Numbers in brackets refer to the bibliography at the end of the paper. 
in $C$, then there exists an element $b \in N, b \notin C$ such that $b^{n}=0$, in which case the theorem would be true. So we may assume that $N \subset C$. Since for every $x \in R, x \notin N$ we can find an $S$ so that $a^{s}-x \in N \subset C$, $\left(a^{s}-x\right) a=a\left(a^{s}-x\right)$; thus $a x=x a$. Also if $x \in N$, since $N \subset C, a x=x a$. Thus $a \in C$. Since $R$ is non-commutative, we can find an $x \notin C$. Thus, since $x \notin N$, for some integer $s, a^{*}-x \in N \subset C$. And so, since $a \in C, a^{\star} \in C$, we conclude that $x \in C$. This is a contradiction; and so we have the theorem.

We are greatly indebted to the referee for pointing out to us the following generalization of the theorem proved above.

THEOREM. Let $R$ be a ring with the property that every element generates a finite subring. Then $R$ is commutative if all the divisors of zero of $R$ are in the centrum.

Proof. Without loss of generality we may assume that every element has characteristic some power of a fixed prime $p$. Let $A$ be the set of divisors of zero of $R$ and $C$ the centrum; by assumption $A \subset C$. If $x \in R, x \notin A$, then $x=x^{r+1}$ for some integer $r>0$; and as $x\left(x^{r} b-b\right)$ $=\left(b x^{r}-b\right) x=0$ for all $b \in R, x^{r}=1$, the identity of $R$. If $A=(0)$ the theorem follows as a corollary of a theorem of Jacobson [4, Theorem 11$, p. 702$]$.

Let us now assume that $A \neq(0)$. If $a \neq 0$ is in $A, c \in R$, then $c a \in A$. Since $A \subset C,(b c-c b) a=0$ for all $b, c \in R, a \in A$. Thus $b c-c b \in A$, and so $(b c-c b)^{2}=0$. For any $b \in R, p b \in A$, so that $p(b c-c b)=0$ for all $b, c \in R$. If $b c-c b=e$, then $b c=c b+e, e \in A, p e=0, e^{2}=0$ and $(b c)^{p}=(c b+e)^{p}=(c b)^{p}$. If for $b, c \in R, b c \in A$, then $b c=c b$. If $b c \notin A$, then $(b c)^{r}=1$ for some $r>0$. Let $r=n p^{k},(n, p)=1$. Then $\left((b c)^{n}-1\right)^{q}$ $=0$ for $q$ some power of $p$. Thus $(b c)^{n}-1 \in A$, and so $(b c)^{n} \in C$. Hence $b(b c)^{n}=(b c)^{n} b$, and so $(b c)^{n}=(c b)^{n}$. As $s n+t p=1, b c=(b c)^{s n}(b c)^{t p}$ $=(c b)^{s n}(c b)^{t p}=c b$, and the theorem follows.

\section{BibliogRAPHY}

1. G. E. Forsythe and N. H. McCoy, On the commutativity of certain rings, Bull. Amer. Math. Soc. vol. 52 (1946) pp. 523-526.

2. H.S. Vandiver, The p-adic representation of rings, Ann. of Math. vol. 48 (1947) pp. 22-28.

3. R. Brauer, On the nilpotency of the radical of a ring, Bull. Amer. Math. Soc. vol. 48 (1942) pp. 752-758.

4. Nathan Jacobson, Structure theory for algebraic algebras, Ann. of Math. vol. 46 (1945) pp. 695-707.

UNIVERSITY OF KANSAS 